\title{
CSF ADA Determination in Early Diagnosis of Tuberculous Meningitis in HIV-Infected Patients
}

\author{
Gopal Chandra Ghosh, ${ }^{1}$ Brijesh Sharma, ${ }^{2}$ and B. B. Gupta ${ }^{2}$ \\ ${ }^{1}$ Hospital Annexe, Christian Medical College, Hospital Campus, Room No. 310, Vellore 632004, India \\ ${ }^{2}$ PGIMER and Dr. RML Hospital, Main Block, New Delhi 100001, India \\ Correspondence should be addressed to Gopal Chandra Ghosh; gcghosh86@gmail.com
}

Received 12 December 2015; Accepted 20 March 2016

Academic Editor: Giuseppe Murdaca

Copyright (c) 2016 Gopal Chandra Ghosh et al. This is an open access article distributed under the Creative Commons Attribution License, which permits unrestricted use, distribution, and reproduction in any medium, provided the original work is properly cited.

Tuberculous and Cryptococcal meningitis are common in HIV patients. A highly specific and sensitive rapid test for diagnosis of Tuberculous meningitis especially in setting of HIV is not available in developing countries where the burden of disease is high. We measured ADA (adenosine deaminase) levels using spectrophotometric method in the CSF of HIV patients with meningitis to differentiate Tuberculous meningitis from meningitis due to other causes. Kruskal-Wallis test was used to compare ADA values between tuberculous meningitis (TBM) and nontuberculous (non-TB) meningitis patients and a receiver-operating characteristic (ROC) analysis curve was drawn from these values. Levels of ADA in the CSF of patients with TBM were significantly higher than those in patients with meningitis due to other causes. CSF ADA level determination with a cut-off value of 6 IU/L was found to be highly specific and fairly sensitive test for the diagnosis of TBM in HIV positive patients.

\section{Introduction}

Tuberculous meningitis (TBM) is an endemic disease in developing countries [1], more so in patients with a low socioeconomic status. TBM remains a major global health problem $[2,3]$

Five lakh patients die of tuberculosis every year in India [4]. The outcome is the worst in multidrug resistant tuberculosis [5]. Studies from India have reported HIV seropositivity rates in patients with tuberculosis to be between 0.4 and $20.1 \%$ [6]. On the other hand, $50 \%$ of HIV-infected patients in India are coinfected with $M$. tuberculosis and approximately 200,000 of these coinfected persons will develop active tuberculosis each year in association with HIV infection [7]. Mortality is significantly higher in HIV-infected patients with tuberculous meningitis $(63.3 \%)$ as compared to HIV negative patients (17.5\%) [8]. Methods of diagnosis of TBM have been found to have low sensitivity and specificity [9].

Newer methods for diagnosing tuberculosis are based on phenotypic and genotypic techniques. For the detection of acid fast bacilli (AFB) in a smear, light microscopy is a common, rapid, and specific method and is used worldwide with a detection rate of $30-40 \%$ [10]. Culture using LowensteinJensen (L-J) medium has sensitivity higher than microscopy but needs several weeks of incubation. A number of genotypic assays based on nucleic acid amplification have been designed including Gen-Probe amplified Mycobacterium tuberculosis direct test, Roche Amplicor MTB test, Cobas Amplicor test, Abbott LCx test, and the BD-Probe Tec (strand displacement amplification) test [11-15]. However, high cost precludes their widespread use in developing countries. A simple and costeffective test for the diagnosis of tuberculous meningitis in HIV positive patients would help to make diagnosis easier.

The definitive criterion for the diagnosis of tuberculous meningitis is demonstration of M. tuberculosis in CSF, by either direct $\mathrm{ZN}$ stained smears or biological culture. However, the sensitivity of CSF ZN staining is $2-87 \%$, and CSF culture is positive for $M$. tuberculosis in $25-75 \%$ of cases [16-22]. Also, routine CSF laboratory findings may not help to establish etiology of meningitis. Thus, CSF ADA levels measurement may be a rapid and important test to differentiate TBM from other causes of meningitis. 
Patients admitted with clinical features suggestive of meningitis (fever, headache, vomiting, and altered consciousness)<smiles>[TeH][TeH]</smiles>

HIV status detection done as per WHO guidelines

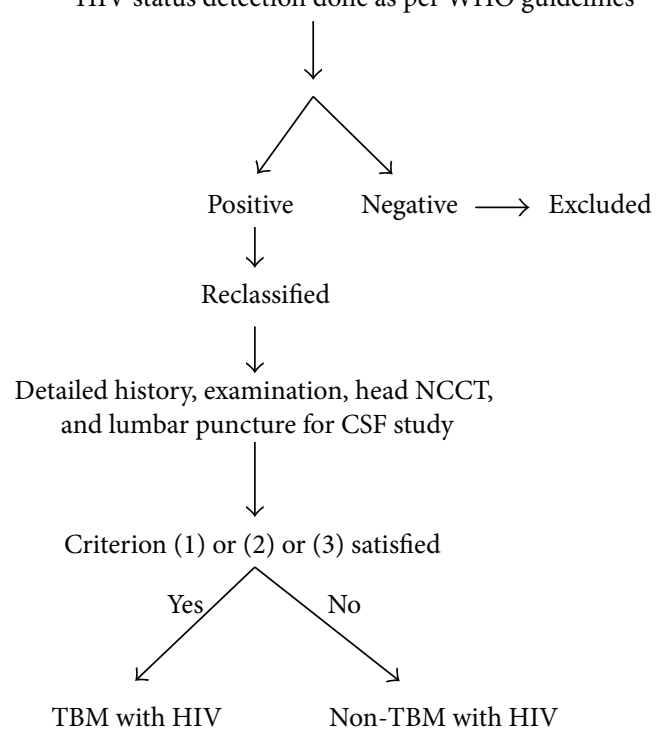

FIGURE 1: Flow chart showing recruitment of patients.

\section{Materials and Methods}

HIV positive patients with meningitis admitted in our department were included in the study. Informed consent was taken from all patients. A detailed history and a clinical examination were performed in all patients. Noncontrast CT scan of the brain was performed followed by lumber puncture under aseptic conditions. A case of tuberculous meningitis was defined as

(1) Mycobacterium tuberculosis detected in CSF by ZiehlNeelsen staining or polymerase chain reaction for Mycobacterium tuberculosis or

(2) tuberculosis at another anatomical site with characteristic clinical and CSF findings or

(3) characteristic CSF findings with improvement after starting antituberculous therapy.

In order to satisfy criterion (2) or (3), India ink staining, latex agglutination test for Cryptococcus, fungal culture, VDRL test, Gram staining, bacterial culture, and latex agglutination test for Streptococcus pneumoniae, Neisseria meningitidis, Haemophilus influenzae, and Listeria monocytogenes in the CSF must be negative. The recruitment and procedure are shown in Figure 1.

ADA levels were estimated in all samples of CSF using the GALANTI and GIUSTI methods.

2.1. Statistical Analysis. ADA levels between patients with tuberculous meningitis and nontuberculous meningitis were compared using the Kruskal-Wallis test. ROC curve analysis was performed to determine the cut-off value for ADA in

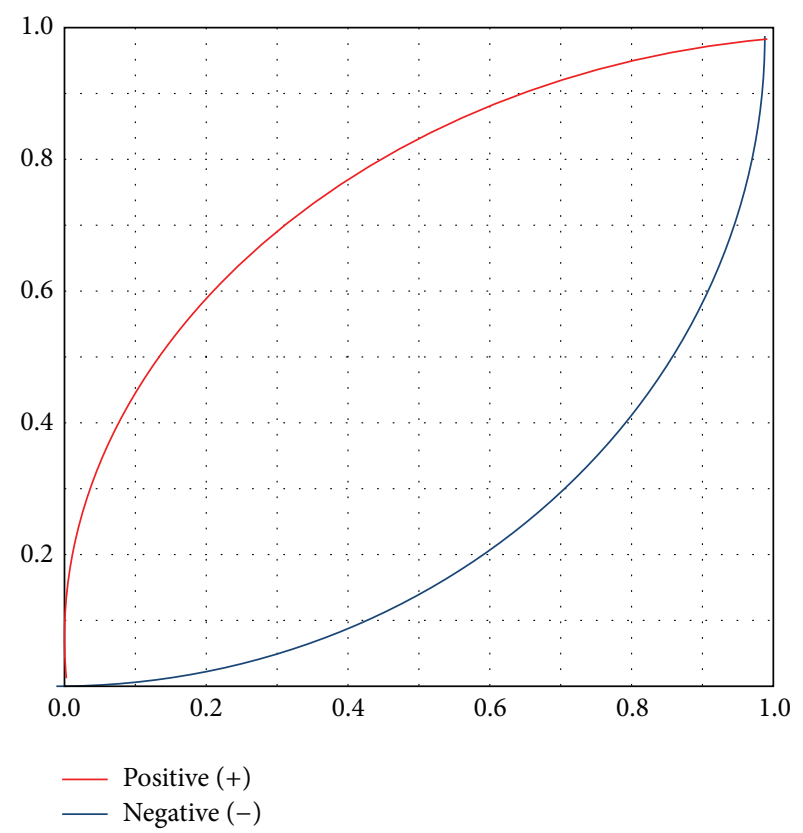

FIgURE 2: Posttest probability graph for the ADA cut-off value of $6 \mathrm{IU} / \mathrm{L}$ in CSF.

order to differentiate TBM from non-TBM groups. Statistical analysis was performed using SPSS version 16. A $P$ value < 0.05 was considered significant.

\section{Results}

We included $40 \mathrm{HIV}$ positive patients with meningitis in our study; of these, 16 were diagnosed as having tuberculous meningitis (TBM) and 24 patients had nontuberculous meningitis (non-TBM). The mean \pm SD age of the patients was $35 \pm 10$ years with the youngest patient being 16 years old and the oldest 65 years old. There were 32 men and 8 women.

Mean \pm SD ADA levels in patients with TBM were 18.1 IU/L $\pm 19.176 \mathrm{IU} / \mathrm{L}$ and mean ADA levels in non-TBM patients were $2.2 \mathrm{IU} / \mathrm{L} \pm 1.8 \mathrm{IU} / \mathrm{L}(P<0.001)$. Posttest probability was high at ADA value of $6 \mathrm{IU} / \mathrm{L}$ and receiveroperating characteristic (ROC) curves analysis of ADA levels in TBM and non-TBM groups revealed area under curve (AUC) of .958 (Figures 2 and 3). A cut-off value of 6 IU/L for CSF ADA activity had sensitivity of $75 \%$, specificity of $95.8 \%$, a positive predictive value of $92.3 \%$, and a negative predictive value of $85.2 \%$ for the diagnosis of TBM.

\section{Discussion and Conclusion}

Determination of ADA levels in CSF is a rapid test to distinguish TBM from non-TB meningitis especially in immunocompetent hosts. Its sensitivity ranges from $75 \%$ to $94 \%$ and specificity from $86 \%$ to $97 \%$ [23-27]. But the role of CSF ADA level determination is still controversial in meningitis patients with HIV as very few studies are available in the literature. A study by Corral et al. [28] found that CSF ADA level cut-off point of $8.5 \mathrm{IU} / \mathrm{L}$ for the diagnosis of tuberculous 


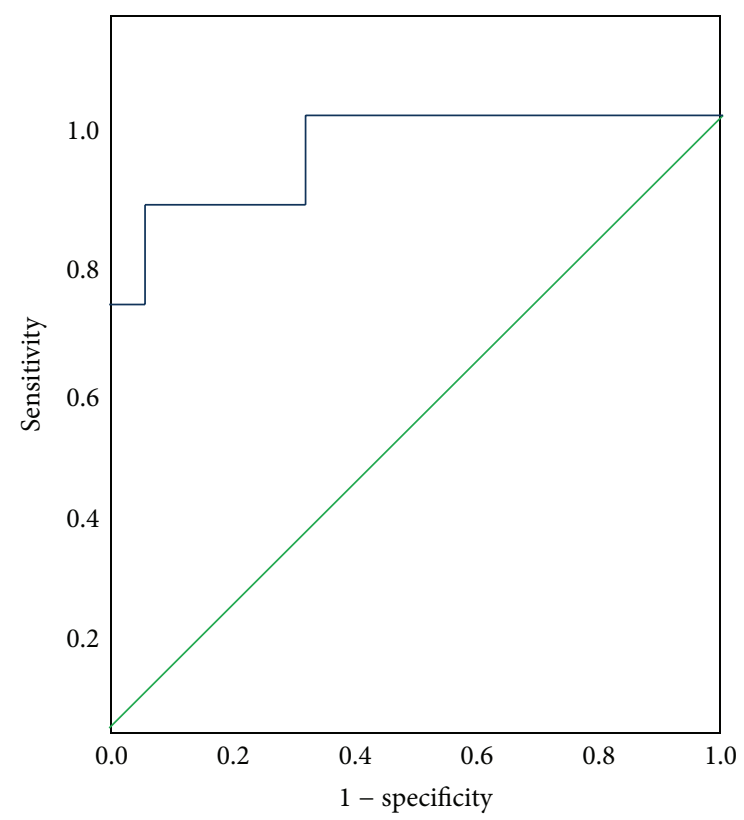

FIGURE 3: The receiver-operating characteristic (ROC) analysis curve for $\mathrm{ADA}$ in tuberculous meningitis and nontuberculous meningitis. Area under curve $(\mathrm{AUC})=.958$.

meningitis had $57 \%$ sensitivity and $87 \%$ specificity; however, another study by Machado et al. [29] found that elevated CSF ADA levels are nonspecific for diagnosis of TBM in HIV positive patients.

We found that a CSF ADA cut-off value of $5 \mathrm{IU} / \mathrm{L}$ has sensitivity of $87.5 \%$ and specificity of $87.5 \%$. On the other hand, a cut-off value of $6 \mathrm{IU} / \mathrm{L}$ has sensitivity of $75 \%$ and specificity of $96.8 \%$ for the diagnosis of TBM in HIV positive patients. High specificity makes this a useful investigation for confirming a diagnosis of TBM in HIV positive patients.

A study by Corral et al. [28] revealed that false-positive results were found in patients with neurological CMV disease and cryptococcal, lymphomatous, and probable candidal meningitis. Patients with HIV associated neurological disorders showed higher ADA levels than patients without neurological disease, but the values were usually within normal ranges. Thus, neither HIV infection per se nor HIV infection of central nervous system causes elevation of ADA levels in cerebrospinal fluid.

In our study a cut-off value of 5 has sensitivity of $87.5 \%$ for diagnosis of tuberculous meningitis but if the cut-off increases to 6 it further increases the specificity to $96.8 \%$, lowering the sensitivity to $75 \%$. But if we consider the cost-effectiveness of the test, disease burden in developing countries, and need of a rapid accurate diagnostic test, then cut-off value of $6 \mathrm{IU} / \mathrm{L}$ has very high specificity and a fair percentage of sensitivity for diagnosis of tuberculous meningitis in HIV positive patients.

Our study result is differing from the study done by Machado et al. This can be because, unlike Machado et al., in our study, none of the patients had candidal meningitis, CMV neurological disease, or neurobrucellosis. On the other hand, our study showed that cryptococcal meningitis is the commonest type of meningitis in HIV positive patients (19 patients). Though the number of cases in our study was small, it demonstrated a statistically significant difference in cerebrospinal fluid ADA levels between tuberculous meningitis and cryptococcal meningitis cases. We need larger studies to further substantiate the role of CSF ADA determination in HIV associated TBM.

\section{Abbreviations \\ WHO: World Health Organisation \\ NCCT: Noncontrast computed tomography scan \\ CSF: Cerebrospinal fluid \\ TBM: Tuberculous meningitis.}

\section{Ethical Approval}

This study was approved by the institutional review board of PGIMER and Dr. Ram Manohar Lohia Hospital, New Delhi.

\section{Competing Interests}

The authors declare that they have no competing interests.

\section{References}

[1] C. M. Mastroianni, F. Paoletti, M. Lichtner, C. D’Agostino, V. Vullo, and S. Delia, "Cerebrospinal fluid cytokines in patients with tuberculous meningitis," Clinical Immunology and Immunopathology, vol. 84, no. 2, pp. 171-176, 1997.

[2] S. Marais, D. J. Pepper, B. J. Marais, and M. E. Török, "HIVassociated tuberculous meningitis-diagnostic and therapeutic challenges," Tuberculosis, vol. 90, no. 6, pp. 367-374, 2010.

[3] P. I. Fujiwara, P. Clevenbergh, and R. A. Dlodlo, "Management of adults living with HIV/AIDS in low-income, high-burden settings, with special reference to persons with tuberculosis," International Journal of Tuberculosis and Lung Disease, vol. 9, no. 9, pp. 946-958, 2005.

[4] P. N. Tandon, "Neurotuberculosis: clinical aspects," in Neurology in Tripica, J. S. Chopra and I. M. S. Sawhney, Eds., pp. 358389, Churchill Levingstone, New York, NY, USA, 1999.

[5] G. Thwaites, T. T. H. Chau, N. T. H. Mai, F. Drobniewski, K. McAdam, and J. Farrar, "Tuberculous meningitis," Journal of Neurology, Neurosurgery \& Psychiatry, vol. 68, no. 3, pp. 289299, 2000.

[6] S. K. Sharma, A. Mohan, and T. Kadhiravan, "HIV-TB coinfection: epidemiology, diagnosis \& management," Indian Journal of Medical Research, vol. 121, no. 4, pp. 550-567, 2005.

[7] G. R. Khatri and T. R. Frieden, "Controlling tuberculosis in India," The New England Journal of Medicine, vol. 347, no. 18, pp. 1420-1425, 2002.

[8] D. Cecchini, J. Ambrosioni, C. Brezzo et al., "Tuberculous meningitis in HIV-infected and non-infected patients: comparison of cerebrospinal fluid findings," International Journal of Tuberculosis and Lung Disease, vol. 13, no. 2, pp. 269-271, 2009.

[9] G. H. Bothamley, "Serological diagnosis of tuberculosis," The European Respiratory Journal. Supplement, vol. 20, pp. 676-688, 1995. 
[10] K. R. Steingart, M. Henry, V. Ng et al., "Fluorescence versus conventional sputum smear microscopy for tuberculosis: a systematic review," Lancet Infectious Diseases, vol. 6, no. 9, pp. 570-581, 2006.

[11] C. Abe, K. Hirano, M. Wada et al., "Detection of Mycobacterium tuberculosis in clinical specimens by polymerase chain reaction and gen-probe amplified mycobacterium tuberculosis direct test," Journal of Clinical Microbiology, vol. 31, no. 12, pp. 32703274, 1993.

[12] K. G. Beavis, M. B. Lichty, D. L. Jungkind, and O. Giger, "Evaluation of Amplicor PCR for direct detection of Mycobacterium tuberculosis from sputum specimens," Journal of Clinical Microbiology, vol. 33, no. 10, pp. 2582-2586, 1995.

[13] J. A. Down, M. A. O'Connell, M. S. Dey et al., "Detection of Mycobacterium tuberculosis in respiratory specimens by strand displacement amplification of DNA," Journal of Clinical Microbiology, vol. 34, no. 4, pp. 860-865, 1996.

[14] J. Lachnik, B. Ackermann, A. Bohrssen et al., "Rapid-cycle PCR and fluorimetry for detection of mycobacteria," Journal of Clinical Microbiology, vol. 40, no. 9, pp. 3364-3373, 2002.

[15] D. Yeboah-Manu, M. D. Yates, and S. M. Wilson, "Application of a simple multiplex PCR to aid in routine work of the Mycobacterium reference laboratory," Journal of Clinical Microbiology, vol. 39, no. 11, pp. 4166-4168, 2001.

[16] D. H. Kennedy and R. J. Fallon, “Tuberculous meningitis," The Journal of the American Medical Association, vol. 241, no. 3, pp. 264-268, 1979.

[17] M. E. Kilpatrick, N. I. Girgis, M. W. Yassin, and A. A. Abu El Ella, "Tuberculous meningitis-clinical and laboratory review of 100 patients," Journal of Hygiene, vol. 96, no. 2, pp. 231-238, 1986.

[18] J. M. Leonard and R. M. Des Prez, "Tuberculous meningitis," Infectious disease clinics of North America, vol. 4, no. 4, pp. 769$787,1990$.

[19] M. Traub, A. C. F. Colchester, D. P. E. Kingsley, and M. Swash, "Tuberculosis of the central nervous system," Quarterly Journal of Medicine, vol. 53, no. 209, pp. 81-100, 1984.

[20] M. P. Dubé, P. D. Holtom, and R. A. Larsen, “Tuberculous meningitis in patients with and without human immunodeficiency virus infection," The American Journal of Medicine, vol. 93, no. 5, pp. 520-524, 1992.

[21] S. J. Kent, S. M. Crowe, A. Yung, C. R. Lucas, and A. M. Mijch, "Tuberculous meningitis: a 30-year review," Clinical Infectious Diseases, vol. 17, no. 6, pp. 987-994, 1993.

[22] R. Verdon, S. Chevret, J.-P. Laissy, and M. Wolff, “Tuberculous meningitis in adults: review of 48 cases," Clinical Infectious Diseases, vol. 22, no. 6, pp. 982-988, 1996.

[23] H. Karsen, S. T. Koruk, M. K. Karahocagil, C. Calisir, and F. C. Baran, "Comparative analysis of cerebrospinal fluid adenosine deaminase activity in meningitis," Swiss Medical Weekly, vol. 141, Article ID 13214, 2011.

[24] B. K. Gupta, A. Bharat, B. Debapriya, and H. Baruaha, "Adenosine deaminase levels in CSF of tuberculous meningitis patients," Journal of Clinical Medicine Research, vol. 2, no. 5, pp. 220-224, 2010.

[25] A. Moghtaderi, A. Niazi, R. Alavi-Naini, S. Yaghoobi, and B. Narouie, "Comparative analysis of cerebrospinal fluid adenosine deaminase in tuberculous and non-tuberculous meningitis," Clinical Neurology and Neurosurgery, vol. 112, no. 6, pp. 459462, 2010.
[26] S. V. Rana, F. Chacko, V. Lal et al., "To compare CSF adenosine deaminase levels and CSF-PCR for tuberculous meningitis," Clinical Neurology and Neurosurgery, vol. 112, no. 5, pp. 424430, 2010.

[27] V. Chotmongkol, Y. Teerajetgul, and C. Yodwut, "Cerebrospinal fluid adenosine deaminase activity for the diagnosis of tuberculous meningitis in adults," Southeast Asian Journal of Tropical Medicine and Public Health, vol. 37, no. 5, pp. 948-952, 2006.

[28] I. Corral, C. Quereda, E. Navas et al., "Adenosine deaminase activity in cerebrospinal fluid of HIV-infected patients: limited value for diagnosis of tuberculous meningitis," European Journal of Clinical Microbiology and Infectious Diseases, vol. 23, no. 6, pp. 471-476, 2004.

[29] L. D. Machado, J. A. Livramento, and A. Spina-França, "Adenosine deaminase in the cerebrospinal fluid of patients with acquired immunodeficiency syndrome," Arquivos de neuropsiquiatria, vol. 53, no. 4, pp. 755-759, 1995. 


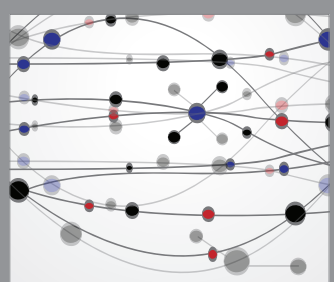

The Scientific World Journal
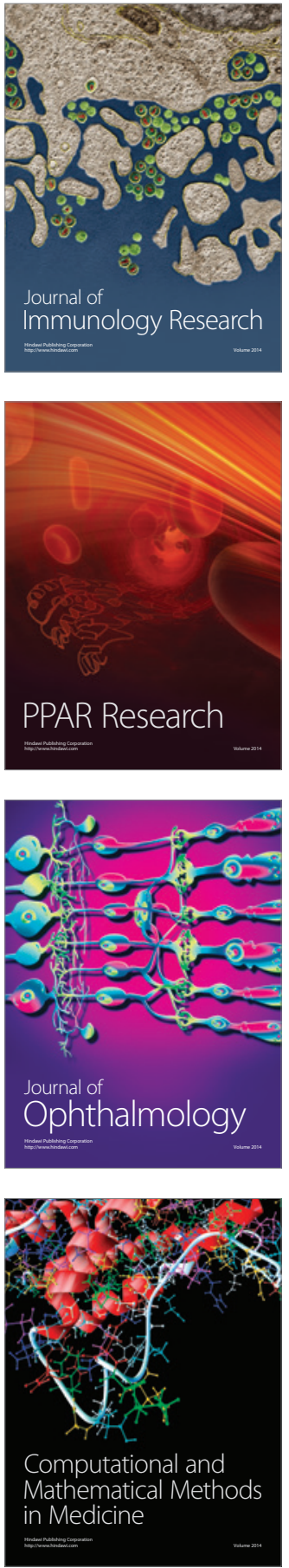

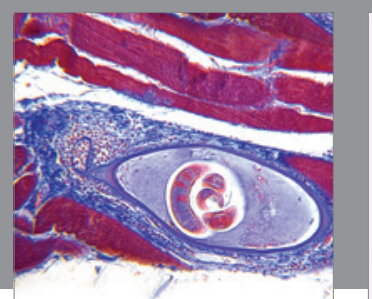

Gastroenterology Research and Practice

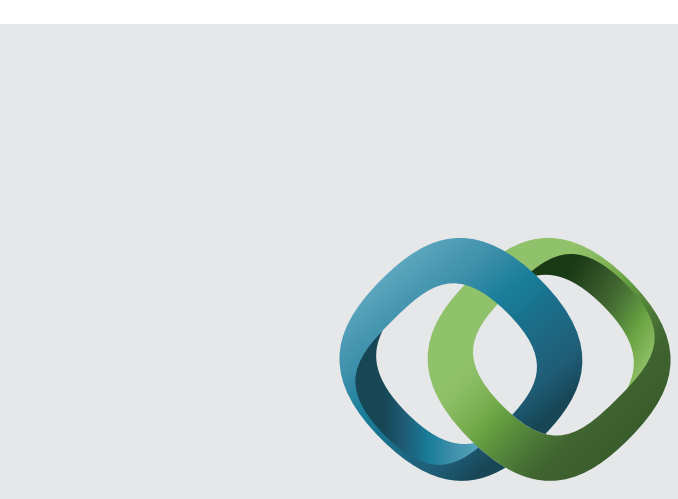

\section{Hindawi}

Submit your manuscripts at

http://www.hindawi.com
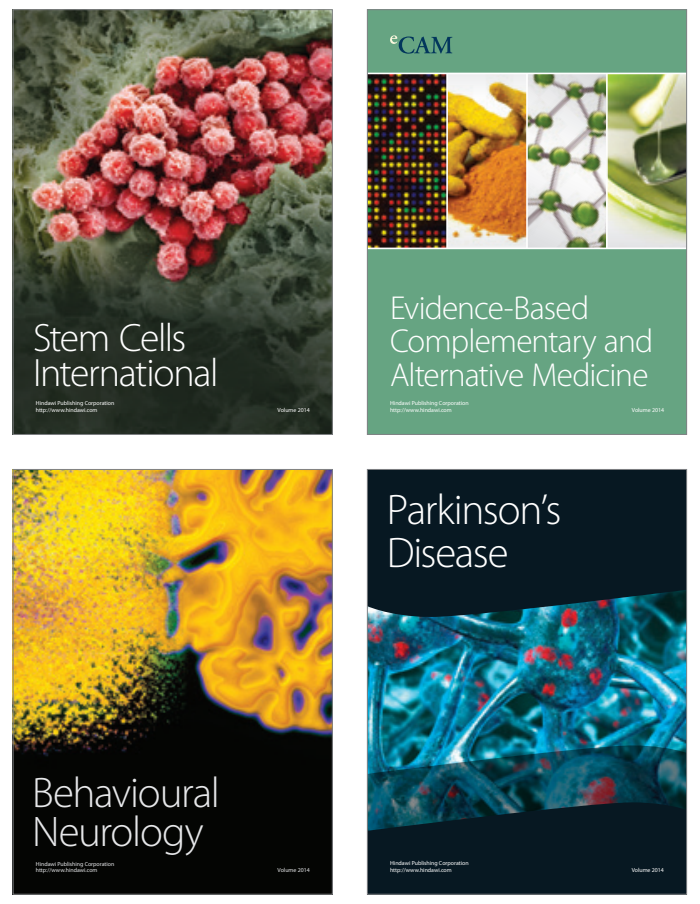
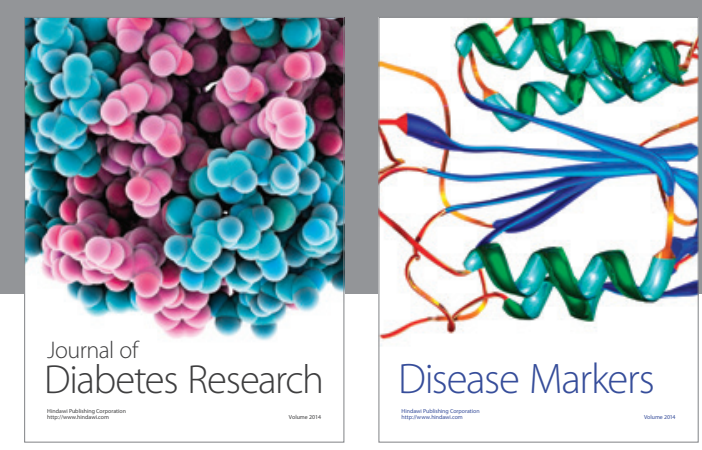

Disease Markers
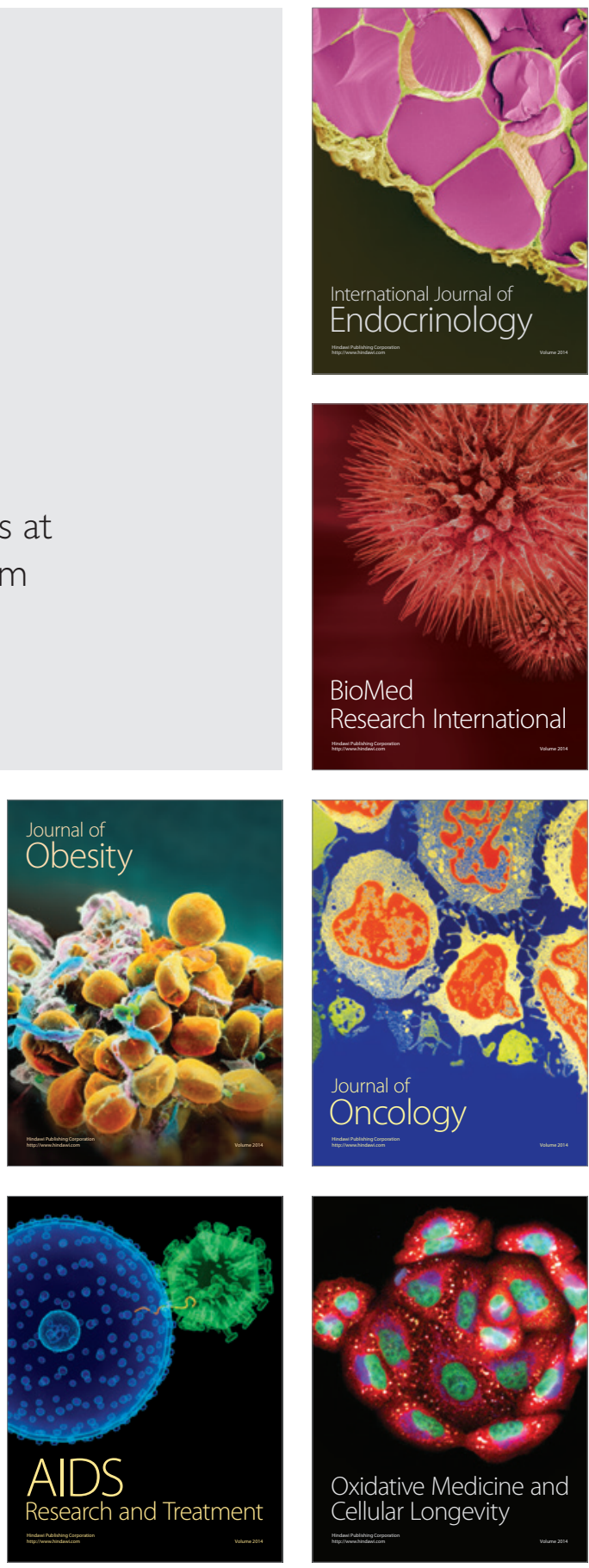